\title{
COVID-19 and Alzheimer's Disease: A Review of Mechanisms and Pathophysiology
}

\author{
Ali Mohammad Khanizadeh ${ }^{1}$, Fariba Karimzadeh ${ }^{2 *}$ \\ ${ }^{1}$ Department of Physiology, School of Medicine, Iran University of Medical Sciences, Tehran, Iran \\ ${ }^{2}$ Cellular and Molecular Research Center, Iran University of Medical Sciences, Tehran, Iran
}

\section{ABSTRACT}

Introduction: SARSCoV2 virus, which has emerged as a worldwide epidemic, is accompanied by systemic symptoms, such as fever, cough, shortness of breath, and body aches. The virus enters the central nervous system in various ways and causes symptoms, such as dizziness, headache, and loss of consciousness, encephalitis, demyelination, neuropathy, stroke, seizure, and memory loss. Infection of the virus into the nervous system, particularly the hippocampus, can cause memory impairment. On the other hand, hypoxia due to lung infection may have a role in the development or progression of Alzheimer's disease (AD). An increase of beta-amyloid production, as well as autophagy following hypoxia, causes nerve damage. Furthermore, chronic hypoxia reduces the expression of beta-amyloid-degrading enzymes by increasing the expression of the beta-secretase enzyme. On the other hand, peripheral proinflammatory cytokines produced by microglia are involved in increasing beta-amyloid levels and tau hyperphosphorylation. Other possible mechanisms involved in the development of AD following the SARSCoV2 virus infection include mitochondrial disorders and increased oxidative stress, which play an important role in the pathophysiology of AD. Oxidative stress increases beta-amyloid production by reducing alpha-secretase activity. Conclusion: The SARSCoV2 virus may exacerbate the symptoms of AD by entering directly into the central nervous system and damaging vital areas in-memory storage or the consequences of chronic hypoxia, oxidative stress, or increased production of peripheral proinflammatory cytokines.

*Corresponding Author: Fariba Karimzadeh

Email: Karimzade.f@iums.ac.ir 
كوويد- 19 و بيمارى آلزايمر: مرورى بر مكانيسم و ياتوفيزيولوزى

$$
\text { على محمد خانىزاده'، فريبا كريمز اده׳" }
$$

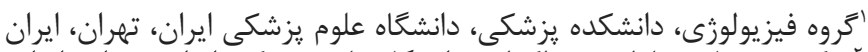

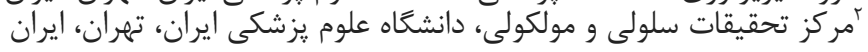

وازههاى كليدى:

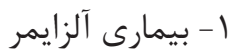

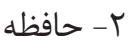
r- استرس اكسيداتيو

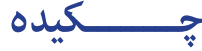

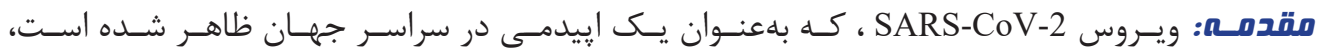

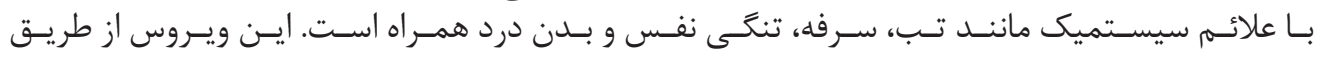

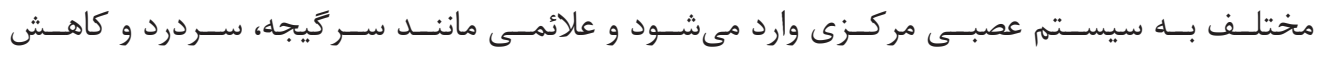

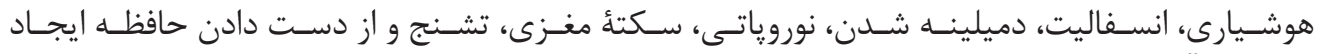

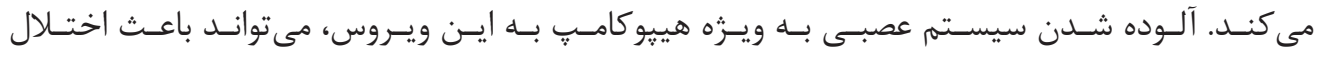

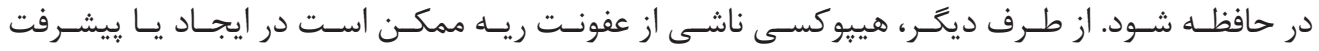

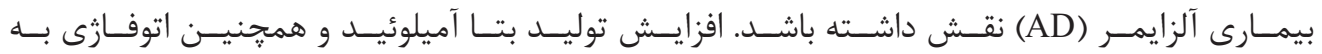

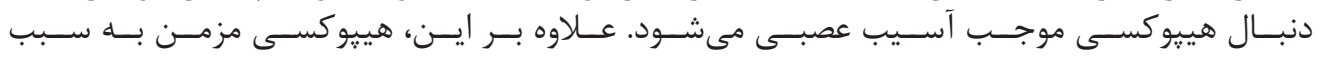

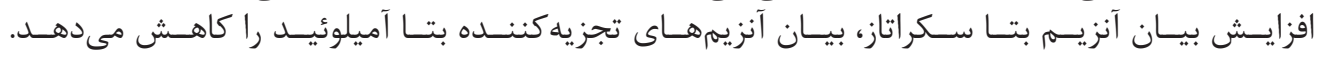

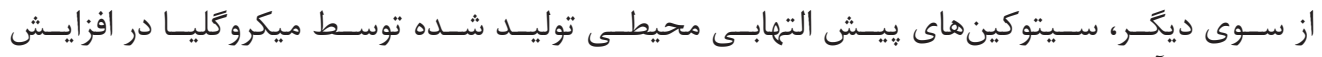

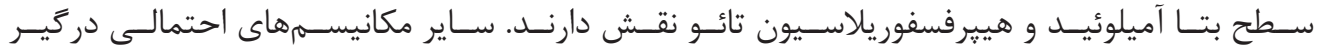

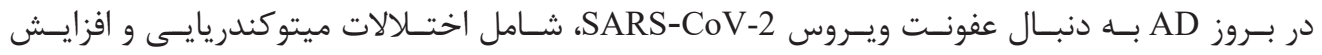

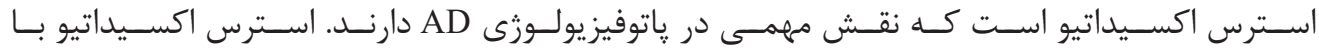

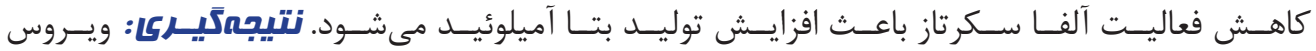
SARS-CoV-2

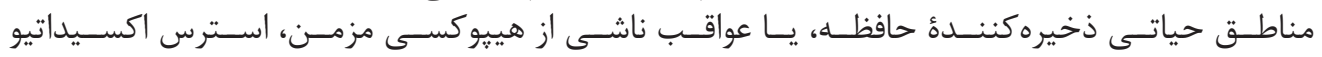

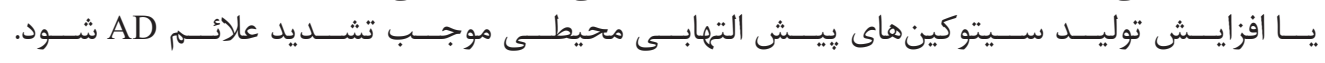


DNA

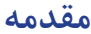

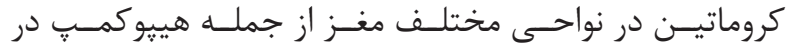

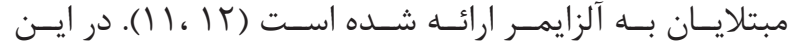

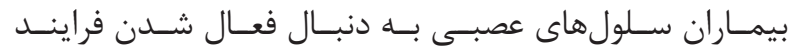

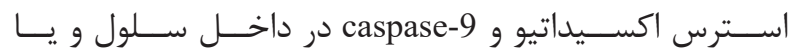

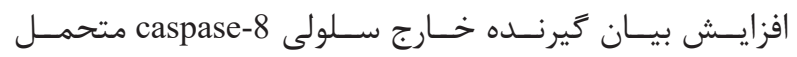

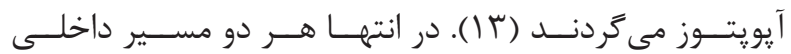

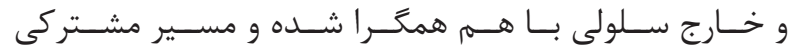

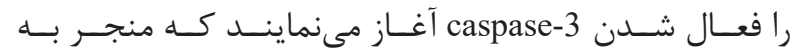

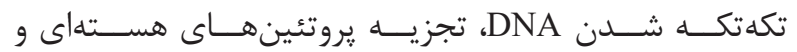

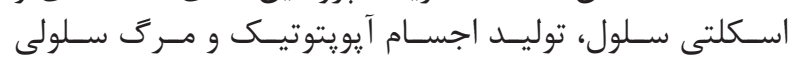

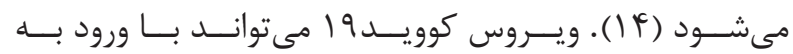

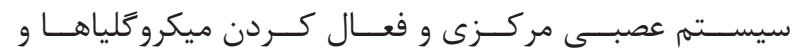

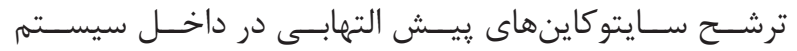

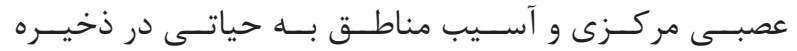

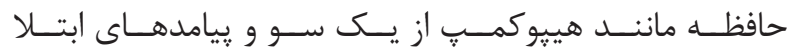

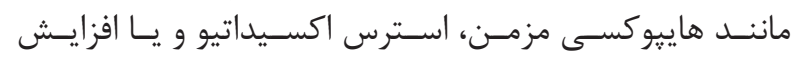

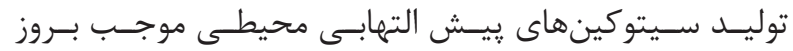

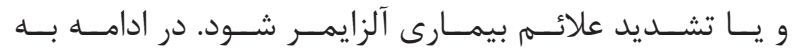

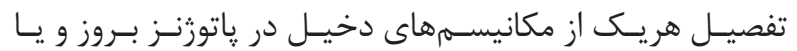

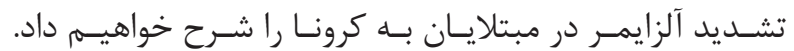

\section{نقش هييوكسى ناشى از ابتلا به كرونا ويروس در آلزايمر}

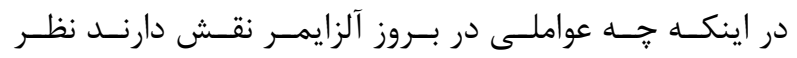

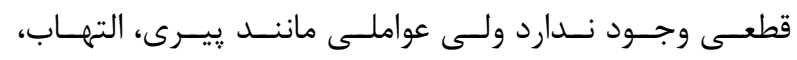

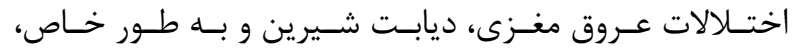

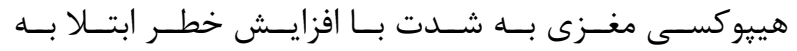

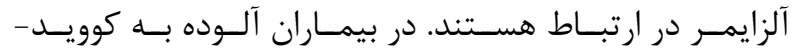

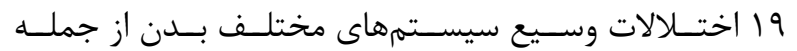

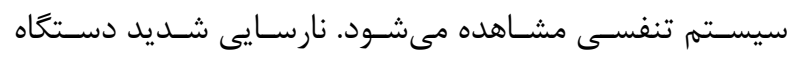

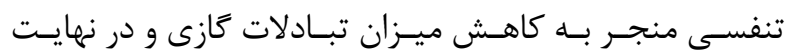

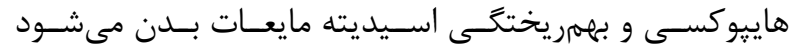

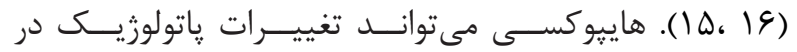

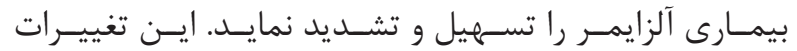

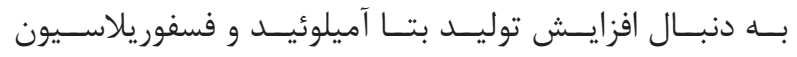

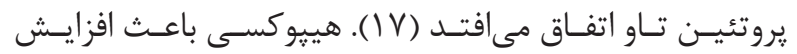

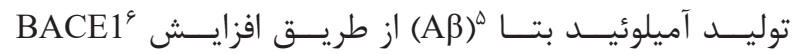

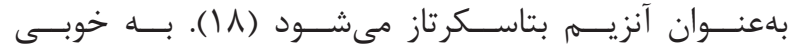

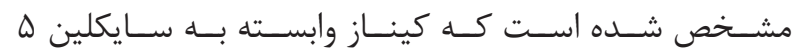

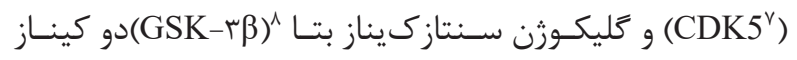

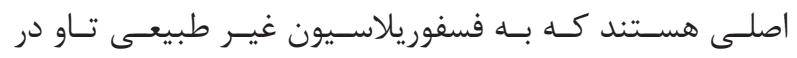

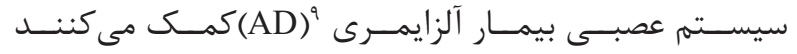

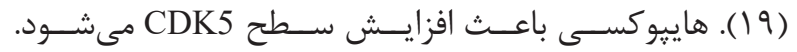

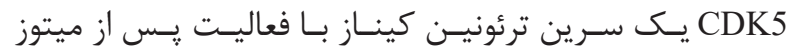

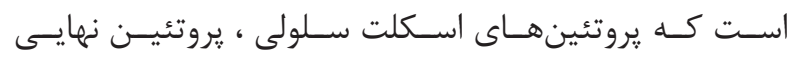

${ }^{1}$ International Committee on Taxonomy of Viruses (ICTV)

${ }^{2}$ Coronavirus Disease-2019

${ }^{3}$ COVID-19

${ }^{4}$ enveloped

${ }^{5}$ Amyloid beta

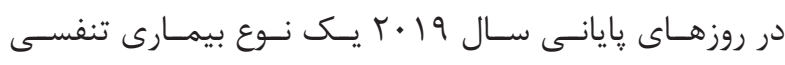

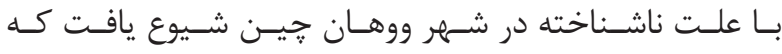

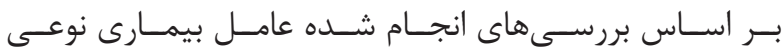

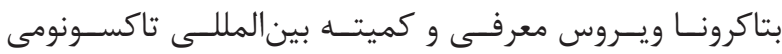

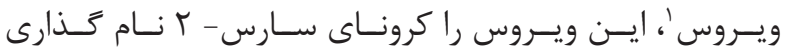

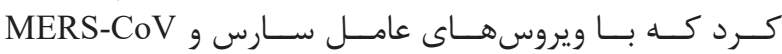

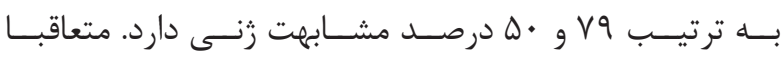

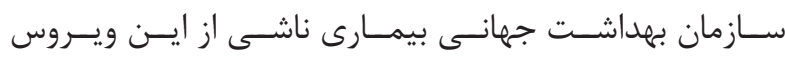

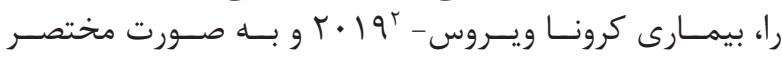

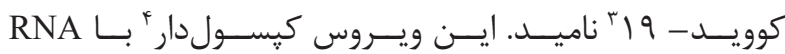

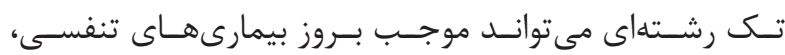

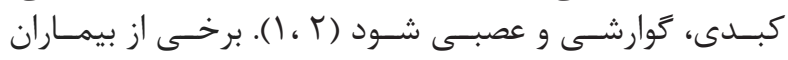

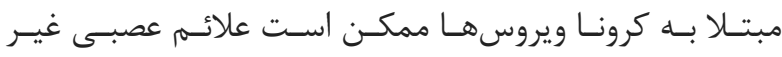

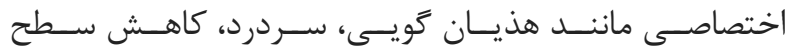

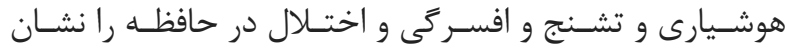

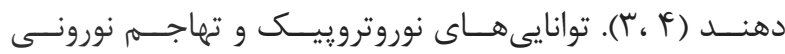

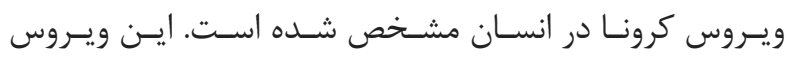

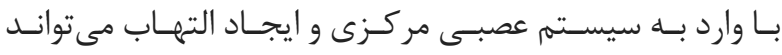

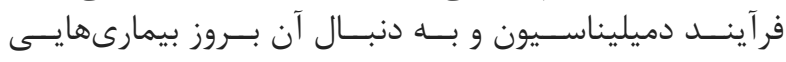

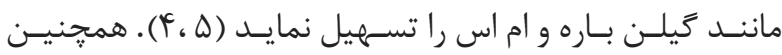

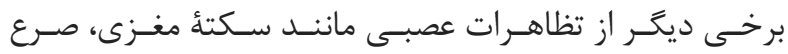

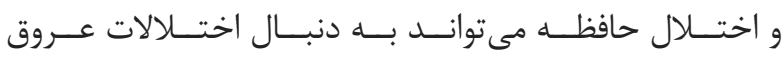

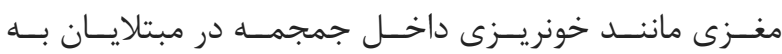

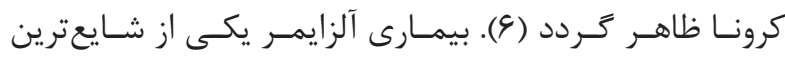

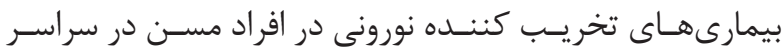

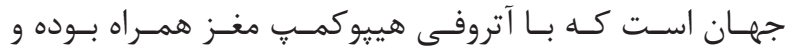

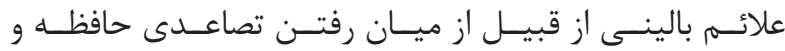

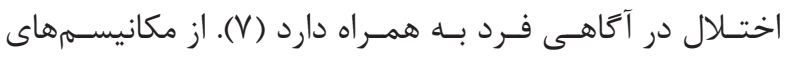

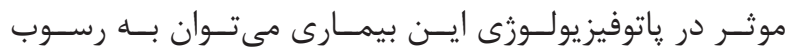

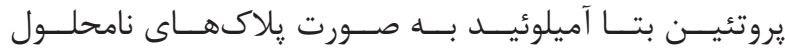

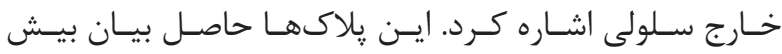

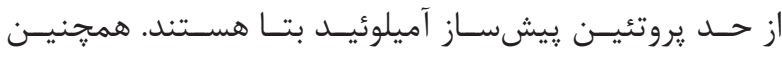

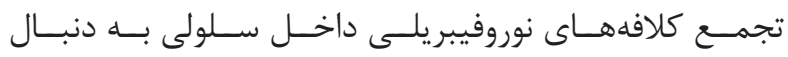

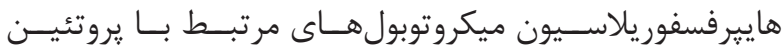
از دau

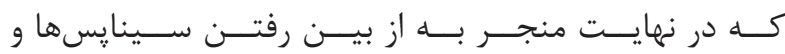

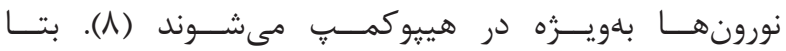

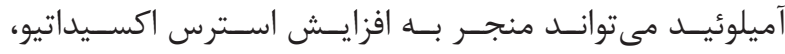

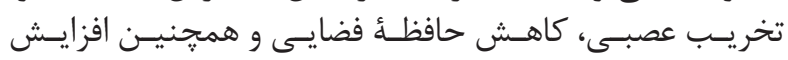

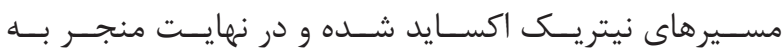

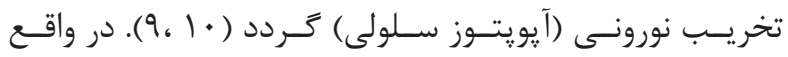

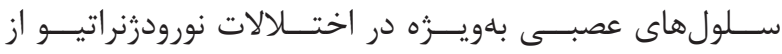

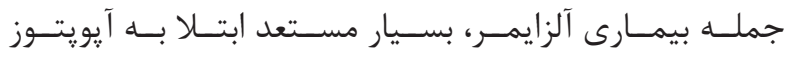

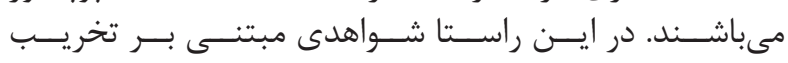

${ }^{6}$ Beta-site amyloid precursor protein-cleaving enzyme 1

${ }^{7}$ Cyclin dependent kinase 5

${ }^{8}$ Glycogen synthase kinase 3 beta

${ }^{9}$ Alzheimer's disease 


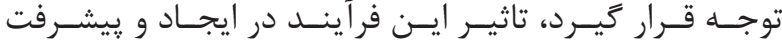

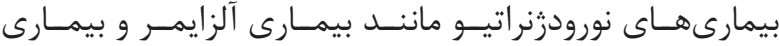

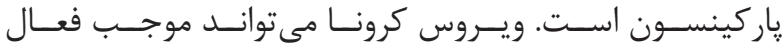

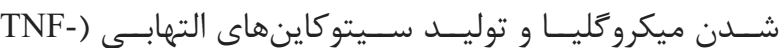

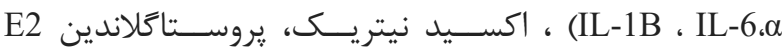

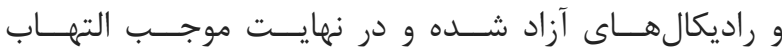

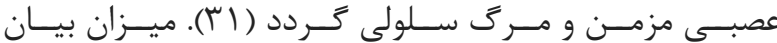

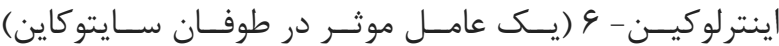

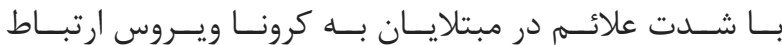

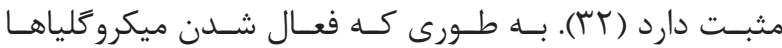

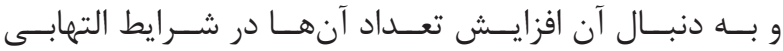

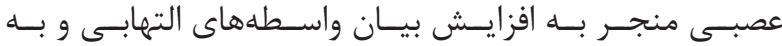

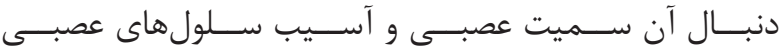

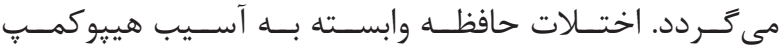

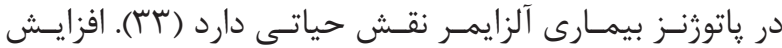

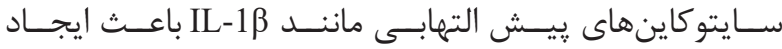

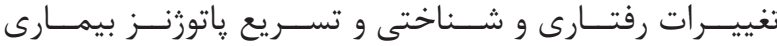

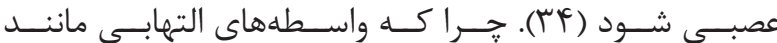

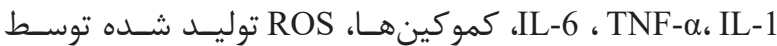

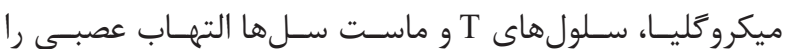

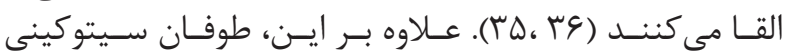

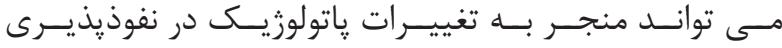

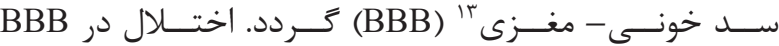

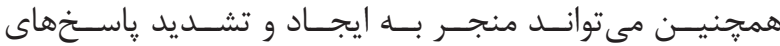

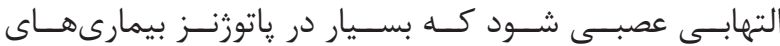

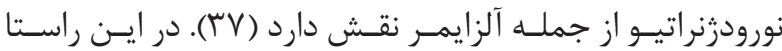

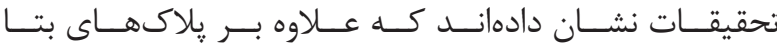

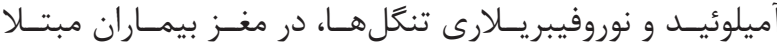

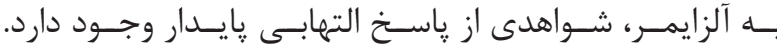

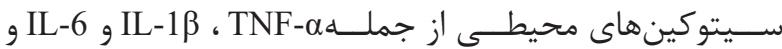
TGF- $\beta$

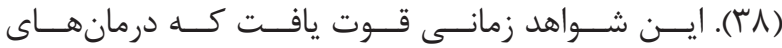

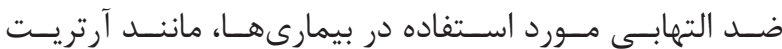

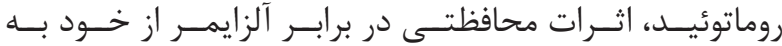

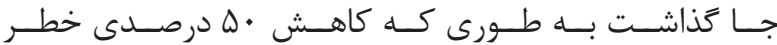

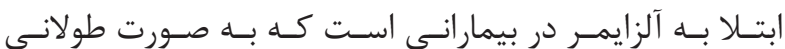

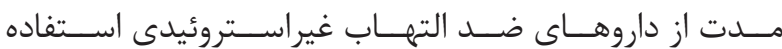

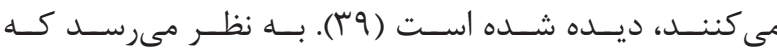

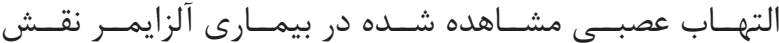

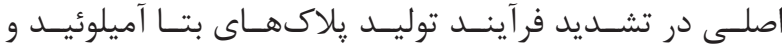

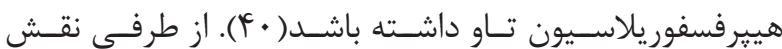

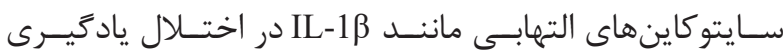

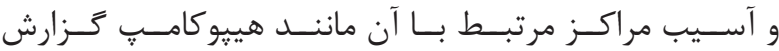

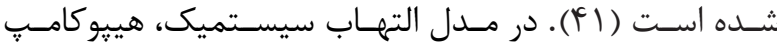

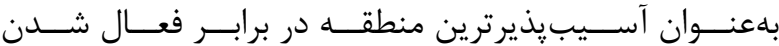

${ }^{10}$ A Distintegrin And Metalloproteinase 10

${ }^{11}$ Autophagic vacuoles

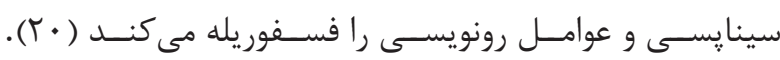

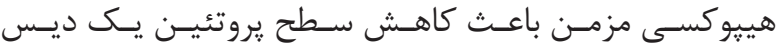

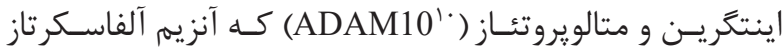

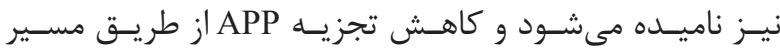

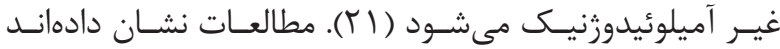

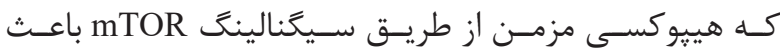

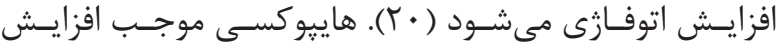

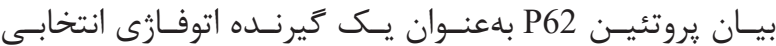

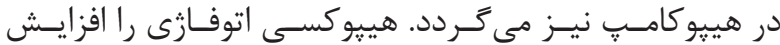

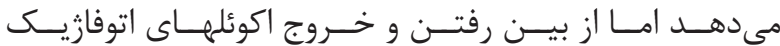

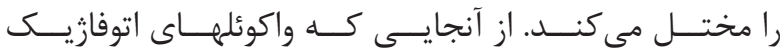

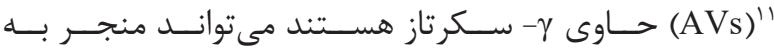

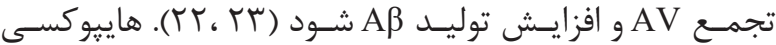

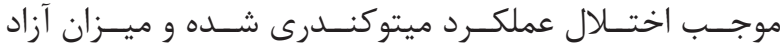

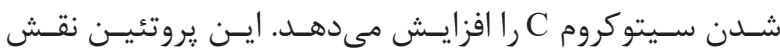

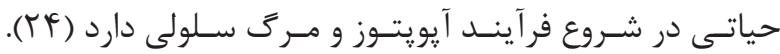

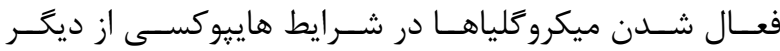

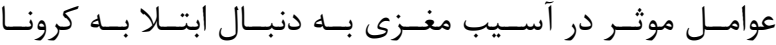

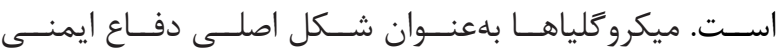

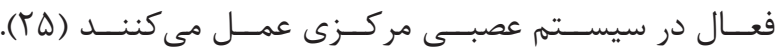

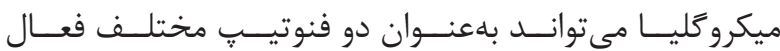

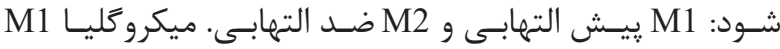

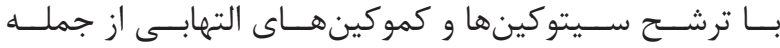
مبـ CCL3 ، CCL2، TNF- $\alpha$ ، IL-6، IL-1

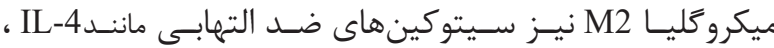

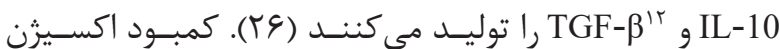

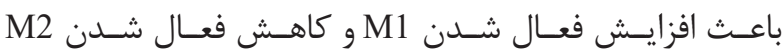

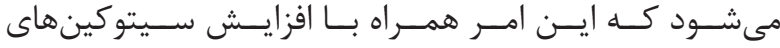

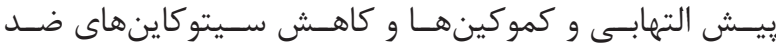

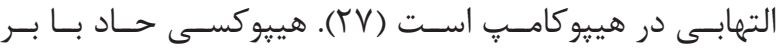

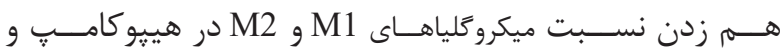

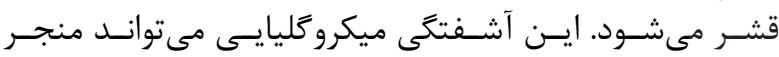

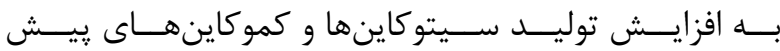

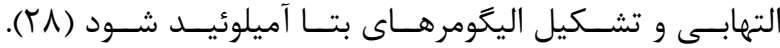

\section{نقش التهاب ناشى از ابتلا به كرونا ويروس در آلزايمر} التهـاب عصبـى يـــ مكانيسـم دفاعسى ميزبــان ذاتسى بـراى

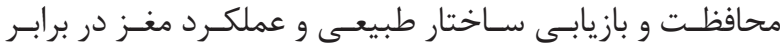

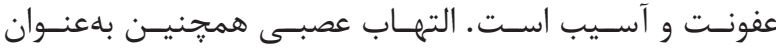

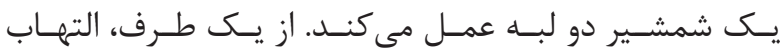

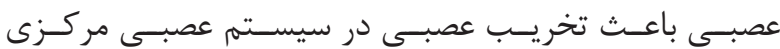

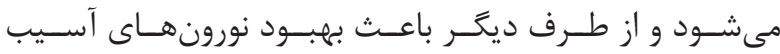

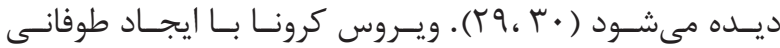

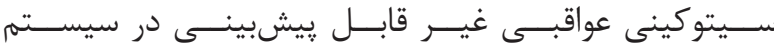

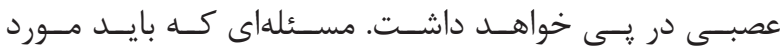

${ }^{12}$ Transforming growth factor beta

${ }^{13}$ Blood-brain barrier 


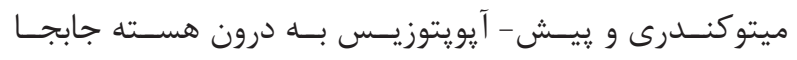

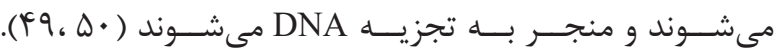

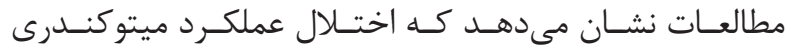

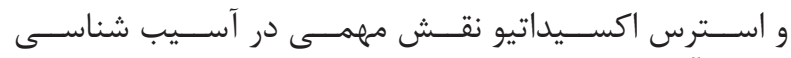

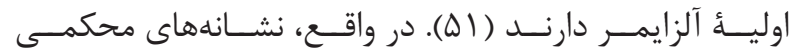

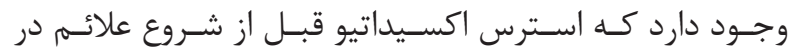

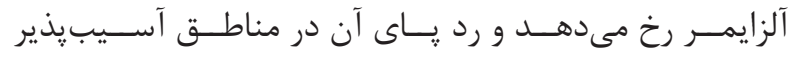

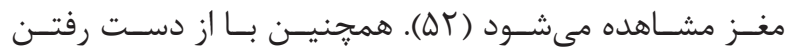

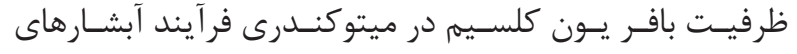

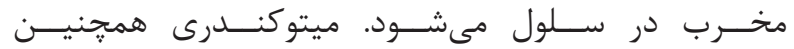

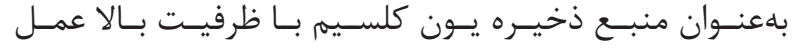

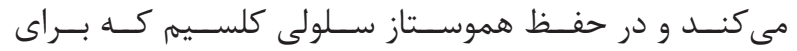

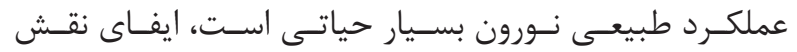

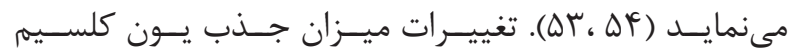

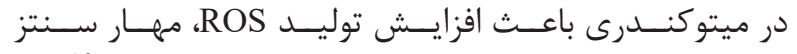

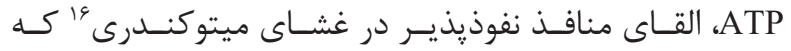

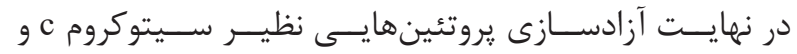

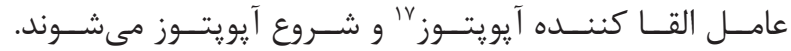

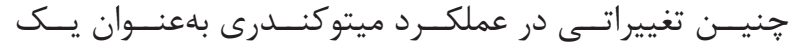

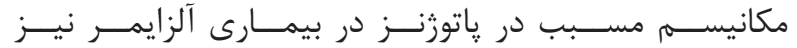

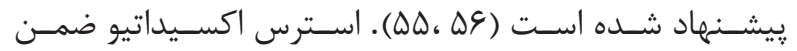

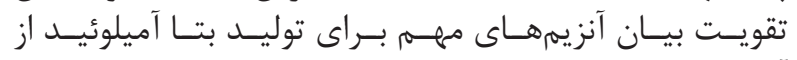

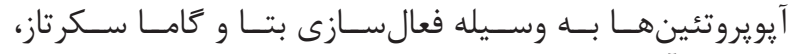

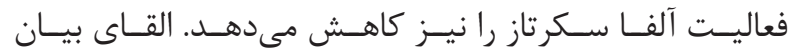

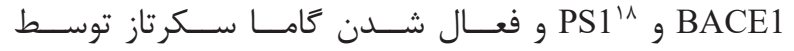

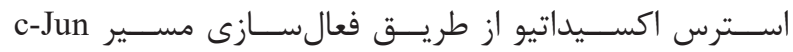

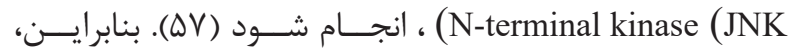

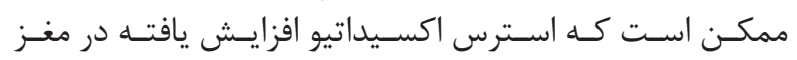

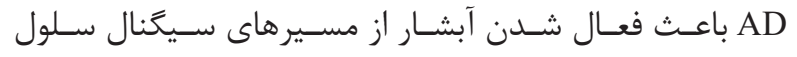

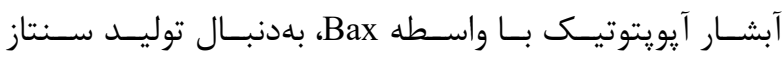

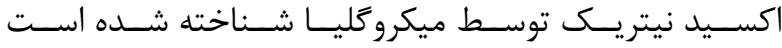

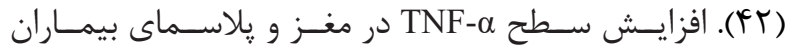

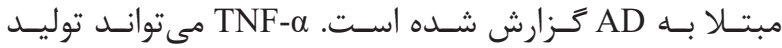

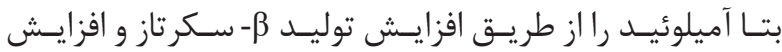

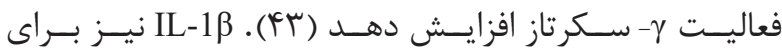

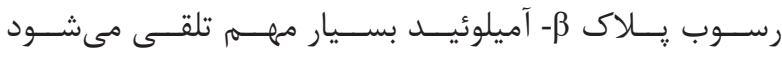

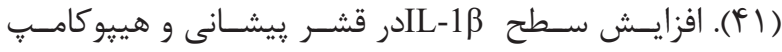

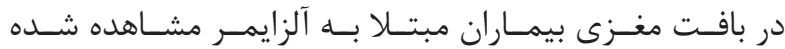

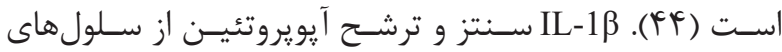

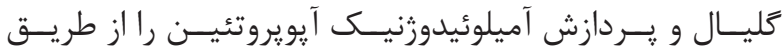

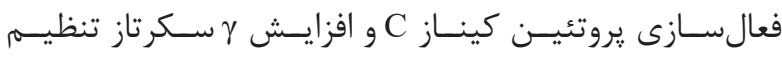

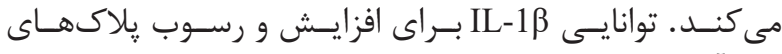

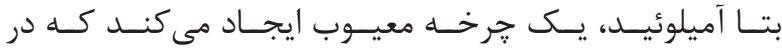

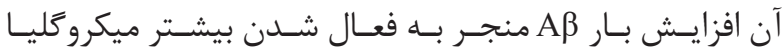

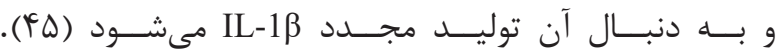

\section{نقش استرس اكسيداتيو ميتوكندرياى به دنبال ابتلا به كرونا ويروس در آلزايمر}

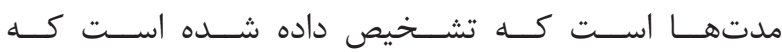

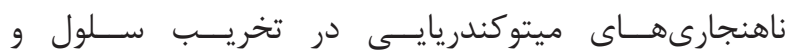

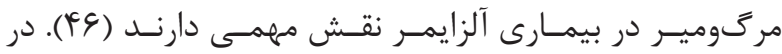

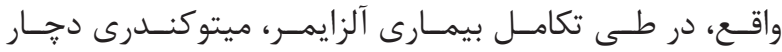

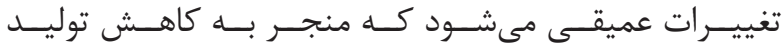

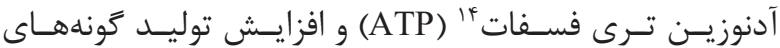

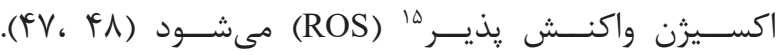

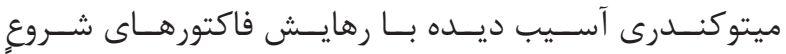

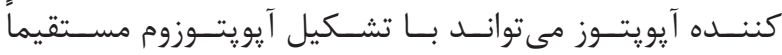

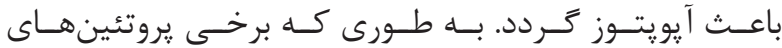

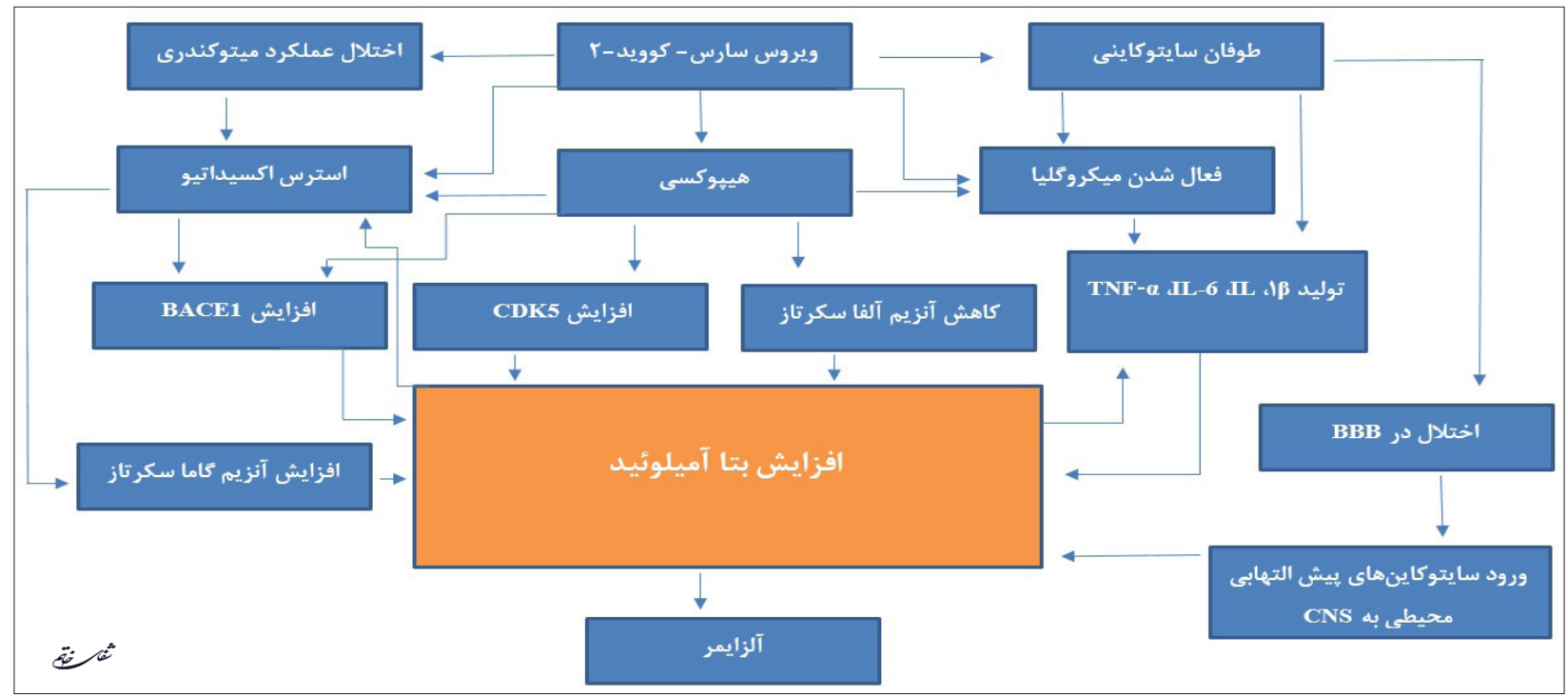

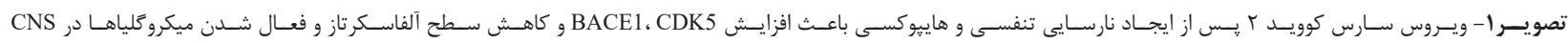

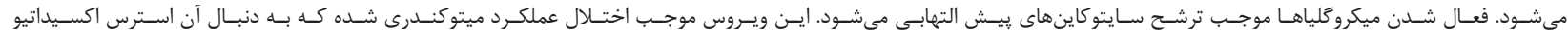

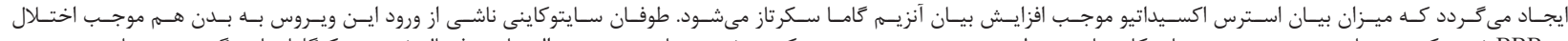

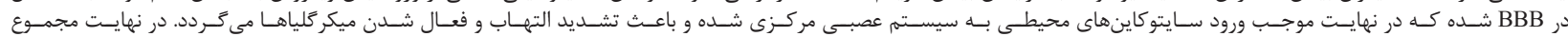

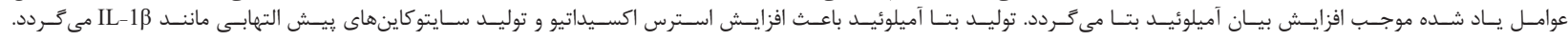

${ }^{14}$ Adenosine triphosphate

${ }^{15}$ Reactive oxygen species

${ }^{16}$ Mitochondrial permeability transition pore
${ }^{17}$ Apoptosis Inducing Factor

${ }^{18}$ Presenilin 1 


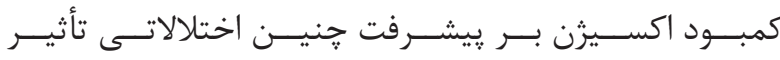

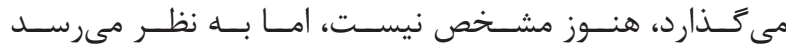

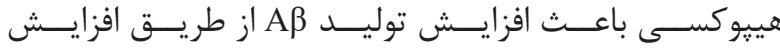

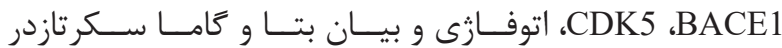

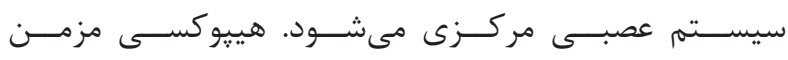

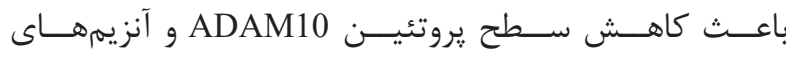

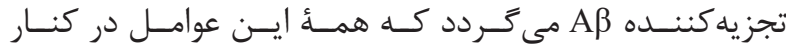

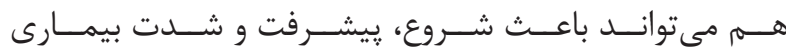

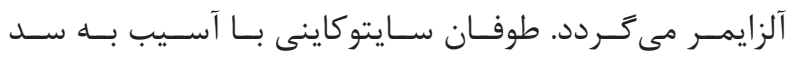

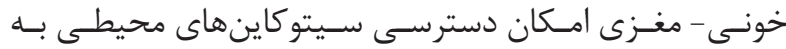

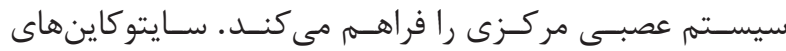

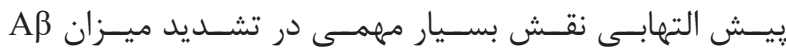

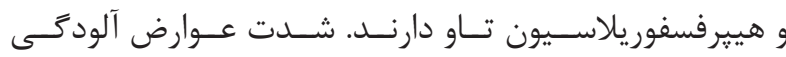

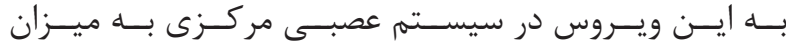

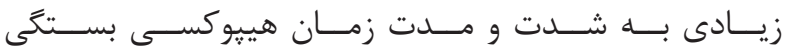

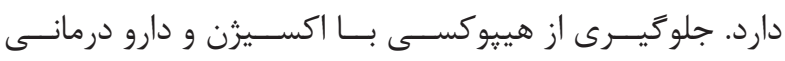

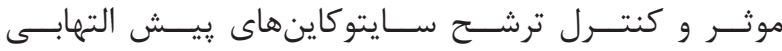

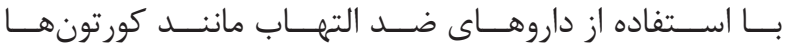

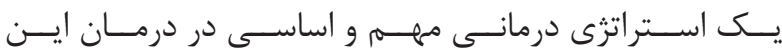

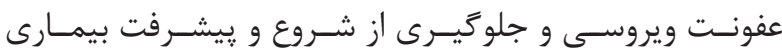

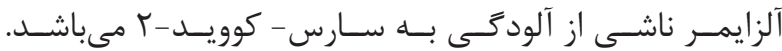

1. Lu H, Stratton CW, Tang YW. Outbreak of pneumonia of unknown etiology in Wuhan, China: the mystery and the miracle. Journal of medical virology. 2020; 92(4): 401-2.

2. Helmy YA, Fawzy M, Elaswad A, Sobieh A, Kenney SP, Shehata AA. The COVID-19 pandemic: a comprehensive review of taxonomy, genetics, epidemiology, diagnosis, treatment, and control. Journal of clinical medicine. 2020; 9(4): 1225.

3. Desforges M, Le Coupanec A, Dubeau P, Bourgouin A, Lajoie L, Dubé M, et al. Human Coronaviruses and Other Respiratory Viruses: Underestimated Opportunistic Pathogens of the Central Nervous System? Viruses. 2020; 12(1): 14.

4. Bohmwald K, Galvez N, Ríos M, Kalergis AM. Neurologic alterations due to respiratory virus infections. Frontiers in cellular neuroscience. 2018; 12: 386.

5. Sedaghat Z, Karimi N. Guillain Barre syndrome associated with COVID-19 infection: a case report. Journal of Clinical Neuroscience. 2020.

6. Asadi-Pooya AA, Simani L. Central nervous system manifestations of COVID-19: A systematic review. Journal of the Neurological Sciences. 2020: 116832.

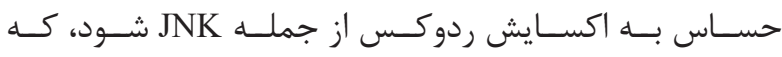

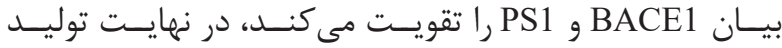

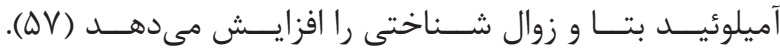
نتيجه كيرى

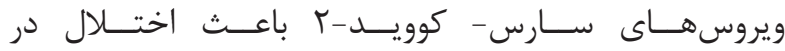

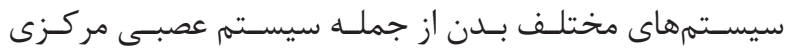

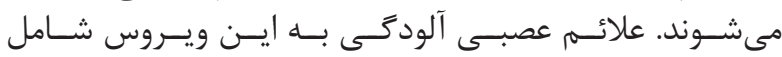

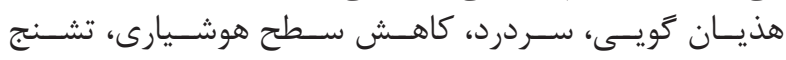

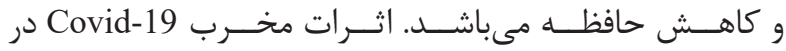

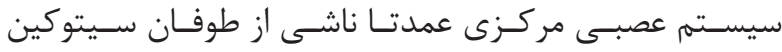

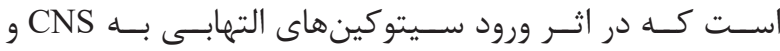

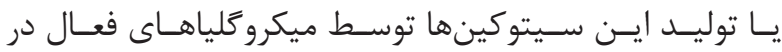

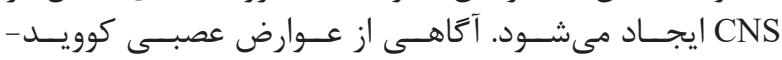

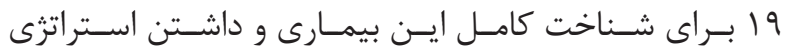

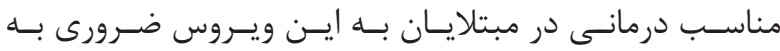

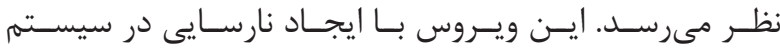

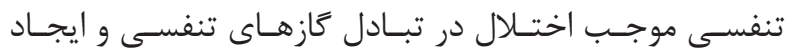

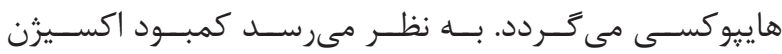

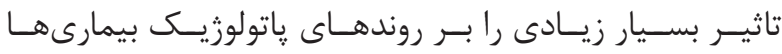

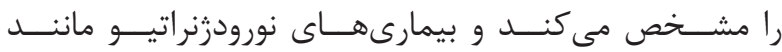

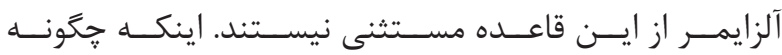

منابع

7. Sinyor B, Mineo J, Ochner C. Alzheimer's Disease, Inflammation, and the Role of Antioxidants. Journal of Alzheimer's Disease Reports. 2020; (Preprint): 1-8.

8. Association As. 2018 Alzheimer's disease facts and figures. Alzheimer's \& Dementia. 2018; 14(3): 367-429.

9. Díaz A, De Jesús L, Mendieta L, Calvillo M, Espinosa $\mathrm{B}$, Zenteno $\mathrm{E}$, et al. The amyloid- $\beta$ 25-35 injection into the CA1 region of the neonatal rat hippocampus impairs the long-term memory because of an increase of nitric oxide. Neuroscience letters. 2010; 468(2): 151-5.

10. Sadeghian H, Jafarian M, Karimzadeh F, Kafami L, Kazemi H, Coulon P, et al. Neuronal death by repetitive cortical spreading depression in juvenile rat brain. Experimental neurology. 2012; 233(1): 438-46.

11. Cotman CW, Su JH. Mechanisms of neuronal death in Alzheimer's disease. Brainpathology.1996;6(4):493-506.

12.JafarianM,KarimzadehF,AlipourF,AttariF,LotfiniaA, Speckmann E-J, et al. Cognitive impairments and neuronal injury in different brain regions of a genetic rat model of absence epilepsy. Neuroscience. 2015; 298: 161-70.

13. Rohn TT, Head E, Nesse WH, Cotman CW, Cribbs 
DH. Activation of caspase- 8 in the Alzheimer's disease brain. Neurobiology of disease. 2001; 8(6): 1006-16.

14. Favaloro B, Allocati N, Graziano V, Di Ilio C, De Laurenzi V. Role of apoptosis in disease. Aging (Albany NY). 2012; 4(5): 330.

15. Couzin-Frankel J. The mystery of the pandemic's 'happy hypoxia'. American Association for the Advancement of Science; 2020.

16. Kashani KB, editor Hypoxia in COVID-19: Sign of Severity or Cause for Poor Outcomes. Mayo Clinic Proceedings; 2020: Mayo Foundation for Medical Education and Research.

17. Liu H, Qiu H, Xiao Q, Le W. Chronic HypoxiaInduced Autophagy Aggravates the Neuropathology of Alzheimer's Disease through AMPK-mTOR Signaling in the APP Swe/PS1 dE9 Mouse Model. Journal of Alzheimer's Disease. 2015; 48(4): 1019-32.

18. Zhang X, Zhou K, Wang R, Cui J, Lipton SA, Liao F-F, et al. Hypoxia-inducible factor $1 \alpha$ (HIF-1 $\alpha$ )-mediated hypoxia increases BACE1 expression and $\beta$-amyloid generation. Journal of Biological Chemistry. 2007; 282(15): 10873-80.

19. Kimura T, Ishiguro K, Hisanaga S-i. Physiological and pathological phosphorylation of tau by Cdk5. Frontiers in molecular neuroscience. 2014; 7: 65.

20. Liu H, Qiu H, Yang J, Ni J, Le W. Chronic hypoxia facilitates Alzheimer's disease through demethylation of $\gamma$-secretase by downregulating DNA methyltransferase 3b. Alzheimer's \& Dementia. 2016; 12(2): 130-43.

21. Rybnikova E, Gluschenko T, Galeeva A, Tulkova E, Nalivaeva NN, Makova NZ, et al. Differential expression of ADAM15 and ADAM17 metalloproteases in the rat brain after severe hypobaric hypoxia and hypoxic preconditioning. Neuroscience research. 2012; 72(4): 364-73.

22. Son SM, Jung ES, Shin HJ, Byun J, MookJung I. A $\beta$-induced formation of autophagosomes is mediated by RAGE-CaMKK $\beta$-AMPK signaling. Neurobiology of aging. 2012; 33(5): 1006. e11-. e23.

23. Neely KM, Green KN, LaFerla FM. Presenilin is necessary for efficient proteolysis through the autophagylysosome system in a $\gamma$-secretase-independent manner.
Journal of Neuroscience. 2011; 31(8): 2781-91.

24. Moreira PI, Carvalho C, Zhu X, Smith MA, Perry $\mathrm{G}$. Mitochondrial dysfunction is a trigger of Alzheimer's disease pathophysiology. Biochimica et Biophysica Acta (BBA)-Molecular Basis of Disease. 2010; 1802(1): 2-10.

25. Saffarzadeh F, Modarres Mousavi SM, Lotfinia AA, Alipour F, Hosseini Ravandi H, Karimzadeh F. Discrepancies of Notch 1 receptor during development of chronic seizures. Journal of cellular physiology. 2019; 234(8): 13773-80.

26. Tang $\mathrm{Y}$, Le W. Differential roles of $\mathrm{M} 1$ and M2 microglia in neurodegenerative diseases. Molecular neurobiology. 2016; 53(2): 1181-94.

27. Zhang F, Zhong R, Li S, Fu Z, Cheng C, Cai H, et al. Acute hypoxia induced an imbalanced M1/M2 activation of microglia through NF- $\kappa \mathrm{B}$ signaling in Alzheimer's disease mice and wild-type littermates. Frontiers in aging neuroscience. 2017; 9: 282.

28. Nakagawa $\mathrm{Y}$, Chiba K. Role of microglial $\mathrm{m} 1 / \mathrm{m} 2$ polarization in relapse and remission of psychiatric disorders and diseases. Pharmaceuticals. $\quad 2014 ; \quad 7(12)$ : $\quad$ 1028-48.

29. Kielian T. Multifaceted roles of neuroinflammation: the need to consider both sides of the coin. Journal of neurochemistry. 2016; 136: 5-9.

30. Stephenson J, Nutma E, van der Valk P, Amor S. Inflammation in CNS neurodegenerative diseases. Immunology. 2018; 154(2): 204-19.

31. Smith JA, Das A, Ray SK, Banik NL. Role of pro-inflammatory cytokines released from microglia in neurodegenerative diseases. Brain research bulletin. 2012; 87(1): 10-20.

32. Wan S, Yi Q, Fan S, Lv J, Zhang X, Guo L, et al. Characteristics of lymphocyte subsets and cytokines in peripheral blood of 123 hospitalized patients with 2019 novel coronavirus pneumonia (NCP). MedRxiv. 2020.

33. Pennisi M, Crupi R, Di Paola R, Ontario ML, Bella R, Calabrese EJ, et al. Inflammasomes, hormesis, and antioxidants in neuroinflammation: role of NRLP3 in Alzheimer disease. Journal of neuroscience research. 2017; 95(7): 1360-72. 
34. Wang RP-H, Ho Y-S, Leung WK, Goto T, Chang RC-C. Systemic inflammation linking chronic periodontitis to cognitive decline. Brain, behavior, and immunity. 2019; 81: 63-73.

35. Komi DEA, Wöhrl S, Bielory L. Mast cell biology at molecular level: a comprehensive review. Clinical reviews in allergy \& immunology. 2019: 1-24.

36.GilfillanAM,AustinSJ,MetcalfeDD.Mastcellbiology: introduction andoverview. MastCellBiology.2011:2-12.

37. Minter MR, Taylor JM, Crack PJ. The contribution of neuroinflammation to amyloid toxicity in Alzheimer's disease. Journal of neurochemistry. 2016;136(3):457-74.

38. Bagyinszky E, Van Giau V, Shim K, Suk K, An SSA, Kim S. Role of inflammatory molecules in the Alzheimer's disease progression and diagnosis. Journal of the neurological sciences. 2017; 376: 242-54.

39. Deardorff WJ, Grossberg GT. Targeting neuroinflammation in Alzheimer's disease: evidence for NSAIDs and novel therapeutics. Expert Review of Neurotherapeutics. 2017; 17(1): 17-32.

40. Walker KA, Ficek BN, Westbrook R. Understanding the role of systemic inflammation in Alzheimer's disease. ACS Publications; 2019.

41. Imamura $Y$, Wang $H$, Matsumoto N, Muroya T, Shimazaki J, Ogura $\mathrm{H}$, et al. Interleukin-1 $\beta$ causes long-term potentiation deficiency in a mouse model of septic encephalopathy. Neuroscience. 2011; 187: 63-9.

42. Semmler A, Okulla T, Sastre M, Dumitrescu-Ozimek L, Heneka MT. Systemic inflammation induces apoptosis with variable vulnerability of different brain regions. Journalofchemicalneuroanatomy.2005;30(2-3):144-57.

43. ChangR, YeeK-L, SumbriaRK. Tumornecrosis factor $\alpha$ inhibition for Alzheimer's disease. Journal of central nervous system disease. 2017; 9: 1179573517709278.

44.DursunE, Gezen-AkD, HanağasıH,BilgiçB,Lohmann E, Ertan S, et al. The interleukin 1 alpha, interleukin 1 beta, interleukin 6 and alpha-2-macroglobulin serum levels in patients with early or late onset Alzheimer's disease, mild cognitive impairment or Parkinson's disease. Journal of neuroimmunology. 2015; 283: 50-7.

45. Rubio-Perez JM, Morillas-Ruiz JM. A review: inflammatory process in Alzheimer's disease, role of cytokines. The Scientific World Journal. 2012; 2012.

46. Islam MT. Oxidative stress and mitochondrial dysfunction-linked neurodegenerative disorders. Neurological research. 2017; 39(1): 73-82.

47. Swerdlow RH. Mitochondria and mitochondrial cascades in Alzheimer's disease. Journal of Alzheimer's Disease. 2018; 62(3): 1403-16.

48. Llanos-González E, Henares-Chavarino ÁA, PedreroPrieto CM, García-Carpintero S, Frontiñán-Rubio J, Sancho-Bielsa FJ, et al. Interplay between mitochondrial oxidative disorders and proteostasis in Alzheimer's disease. Frontiers in Neuroscience. 2020; 13: 1444.

49. Khodaie B, Lotfinia AA, Ahmadi M, Lotfinia M, Jafarian M, Karimzadeh F, et al. Structural and functional effects of social isolation on the hippocampus of rats with traumatic brain injury. Behavioural brain research. 2015; 278: 55-65.

50. Karimzadeh F, Soleimani M, Mehdizadeh M, Jafarian M, Mohamadpour M, Kazemi H, et al. Diminution of the NMDA receptor NR2B subunit in cortical and subcortical areas of WAG/Rij rats. Synapse. 2013; 67(12): 839-46.

51. Moreira PI, Duarte AI, Santos MS, Rego AC, Oliveira CR. An integrative view of the role of oxidative stress, mitochondria and insulin in Alzheimer's disease. Journal of Alzheimer's Disease. 2009; 16(4): 741-61.

52. Kowalska M, Wize K, Prendecki M, Lianeri M, Kozubski W, Dorszewska J. Genetic variants and oxidative stress in Alzheimer's disease. Current Alzheimer Research. 2020; 17(3): 208-23.

53. Calvo-Rodriguez M, Hou SS, Snyder AC, Kharitonova EK, Russ AN, Das $\mathrm{S}$, et al. Increased mitochondrial calcium levels associated with neuronal death in a mouse model of Alzheimer's disease. Nature Communications. 2020; 11(1): 1-17.

54. MüllerM, Ahumada-Castro U, Sanhueza M, GonzalezBillault C, Cárdenas C. Mitochondria and calcium regulation as basis of neurodegeneration associated with aging. Frontiers in Neuroscience. 2018; 12: 470.

55. Wang W, Zhao F, Ma X, Perry G, Zhu X. Mitochondria dysfunction in the pathogenesis of Alzheimer's disease: recent advances. 
Molecular Neurodegeneration. 2020; 15: 1-22. 57. Zhao Y, Zhao B. Oxidative stress and the

56. Agrawal I, Jha S. Mitochondrial dysfunction and Alzheimer's disease: Role of microglia. pathogenesis of Alzheimer's disease. Oxidative medicine and cellular longevity. 2013; 2013.

Frontiers in Aging Neuroscience. 2020; 12: 252. 